\title{
Foam drainage in two dimensions
}

\author{
S. Hutzler, S.J. Cox and G. Wang \\ Physics Department, Trinity College, Dublin 2, Ireland
}

\begin{abstract}
A two-dimensional foam between glass plates is subjected to a forced drainage experiment. The propagation of the surfactant solution into the foam is measured and compared with computer simulations of a foam drainage equation. The spread of liquid both vertically, due to gravity, and horizontally, due to capillary suction, is tracked over time. Finally, the effect of varying the width of the input pipe is investigated: the velocities of the wetting fronts are shown to strongly depend on the ratio of the width of the input to the width of the cell.
\end{abstract}

Key words: Foam Drainage, Simulation, Experiment

\section{Introduction}

Foam drainage is the flow of liquid through a foam, driven by gravity and capillarity, and is an important aspect of the physics of foams [1]. It is ultimately linked to foam stability, which is of great industrial relevance, for example in the brewing and petroleum industries. The influence that drainage has on the variations of density within a foam sample has recently been highlighted for metallic foams, used for example in the automobile industry where homogeneity is of paramount importance [2].

Theories of foam drainage have made great progress over the last ten years [3,4], although the work of Lemlich and co-workers in the 1960s deserves special mention [5]. The performance of forced drainage experiments in which surfactant solution is injected into a foam at a constant flow-rate, followed by local measurements of the liquid fraction, were crucial for this development.

However, experiments and theory have mainly been focused on drainage in one dimension, i.e. in (mainly cylindrical) geometries for which the input flow covers the whole foam [1]. Only a limited 
amount of work concerns two-dimensional flow, notable examples being the study of the spreading of a pulse of solution [6] and the drainage through a foam in a trapezoidal container [7].

In this paper we address the issue of foam drainage in two dimensions by performing both experiments and simulations of forced drainage for foams contained in a rectangular Hele-Shaw cell. Our aim is to prove the predictive capacity of the drainage equations in this geometry, so that we can be sure of our understanding of the balance between gravitational and capillary effects, which is not apparent from a one-dimensional experiment.

Figure 1 shows our experimental set-up. We have added food dye to the surfactant solution used to make the foam and injected the same liquid. This allows for a determination of the local liquid fraction from digitized images. Figure 2 shows that the draining liquid not only moves downwards as a result of gravitational forces but also spreads in the horizontal direction due to capillary forces. Varying the ratio of input width to container width plays a crucial role in the spread of the drainage wave. All of this can be well understood, at least qualitatively, by solving the two-dimensional drainage equation numerically.

\section{Experimental set-up and technique}

Our Hele-Shaw cell consisted of two parallel glass plates (height $H=14 \mathrm{~cm}$, half-width $W=$ $6 \mathrm{~cm}$ ) separated by a depth of $D=3.1 \mathrm{~mm}$, as indicated in figure 1 . The sides of the cell are sealed, so that liquid may only enter at the top and bottom. In order to visualise the drainage process we added 10 parts dark green food colouring to 1 part detergent (Fairy liquid) and 40 parts water. The cell was filled by bubbling nitrogen into this solution: a constant gas flow-rate resulted in a monodisperse foam with a bubble diameter of $d=1 \mathrm{~mm}$.

In a forced drainage experiment the same solution is added to the top of the foam through a needle at a constant flow-rate. The resulting variation of the foam colour (shades of green) is then monitored by taking pictures with a digital camera, situated in front of the foam cell. To vary the width $w$ of the input pipe, we arrange several needles in a row, all supplied from the same source.

Relating the grey values of the digital images to the local liquid fraction requires calibration measurements. These consist of one-dimensional forced drainage experiments in a cell with a square cross-section of area $A_{c}=15 \mathrm{~mm}^{2}$, which was filled with gas bubbles of the same diameter, $d=1 \mathrm{~mm}$, as above. The resulting drainage wave moves only in the vertical direction with velocity $v$ which can be determined easily from the profiles of liquid fraction against vertical position over time. Once this wave has reached the bottom of the foam column, the liquid fraction $\Phi_{l}$ is constant 


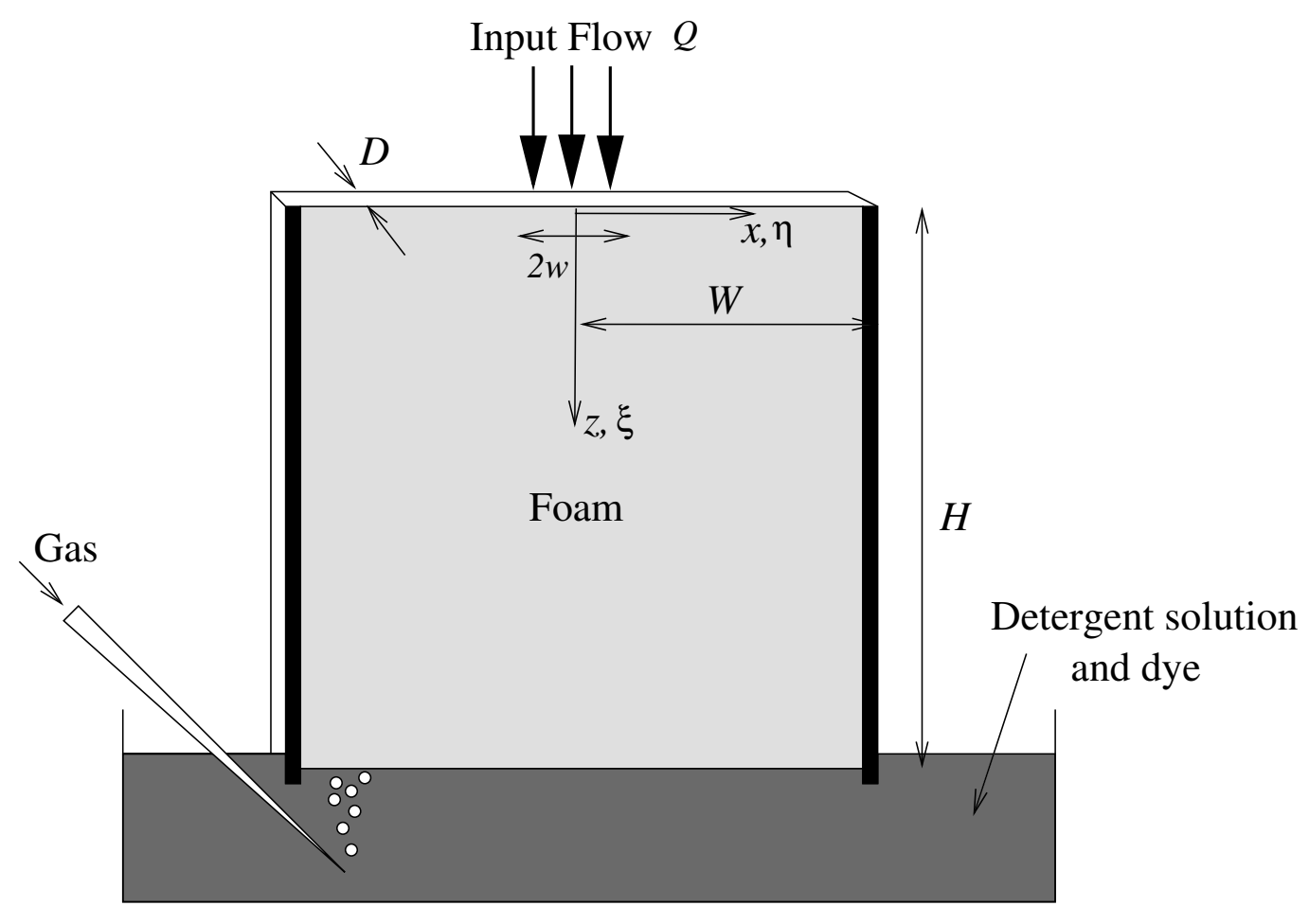

Fig. 1. Experimental set-up for a two dimensional forced drainage experiment. The foaming solution consisted of dishwashing detergent, food colouring (dye) and water. A supply of Nitrogen gas at constant flow-rate resulted in a monodisperse foam between two parallel glass plates. The dimensions of the plates were $W=6 \mathrm{~cm}, H=14 \mathrm{~cm}$ and $D=0.31 \mathrm{~cm}$. The bubble diameter was $d=1 \mathrm{~mm}$. We varied the input flow-rate $Q$ and the width $w$ of the input pipe, and describe the resulting drainage wave in terms of coordinates $x$ and $z$.

throughout and is obtained from

$$
Q=\Phi_{l} A_{c} v
$$

where $Q$ is the total volumetric flow-rate of added solution.

Figure 3 shows how the experimentally determined liquid fraction $\Phi_{l}$ varies with the measured light intensity, as represented by the grey value of the digital images. We have used this relationship to transform the grey-scale of a digitised image of a two-dimensional drainage experiment into an image showing local liquid fraction. 


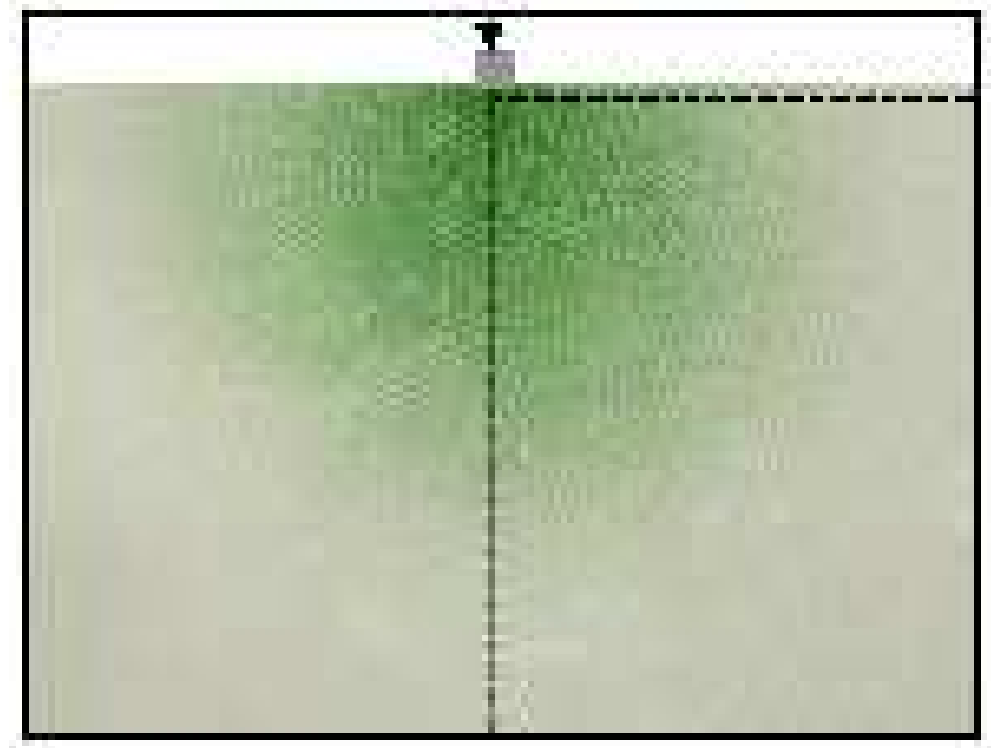

Fig. 2. Forced drainage using a narrow input. Darker foam corresponds to a higher value of local liquid fraction. The photograph was taken about $14 \mathrm{~s}$ after the commencement of forced drainage from the top of a dry foam with a constant flow-rate of solution. As the draining liquid moves downwards (as a result of gravitational forces) it also spreads horizontally (due to capillary forces). We measure the liquid fraction profiles along the vertical and horizontal dotted lines indicated.

\section{The two-dimensional drainage equation}

Standard drainage theories assume that the flow of liquid proceeds through the network of Plateau borders only, i.e the flow through films may be neglected. Two limiting cases have been identified, depending upon where the viscous dissipation occurs: the liquid in the Plateau borders may experience either Poiseuille flow (no-slip condition) or plug flow (with dissipation in the Plateau border junctions). In each case, corresponding drainage equations can be set up [3,4]. In any experiment the condition of flow is somewhere between these two limits, depending on the surface viscosity of the surfactant in use [8]: high surface viscosity leads to Poiseuille flow and vice versa.

In our experiments we have used the detergent Fairy Liquid which has been shown to be well described by the Poiseuille flow limit [9]. In this case the relevant drainage equation is given by [10]:

$$
150 \mu \frac{\partial A}{\partial t}+\rho g \frac{\partial A^{2}}{\partial z}-C \gamma \frac{1}{3} \nabla^{2} A^{3 / 2}=0 .
$$




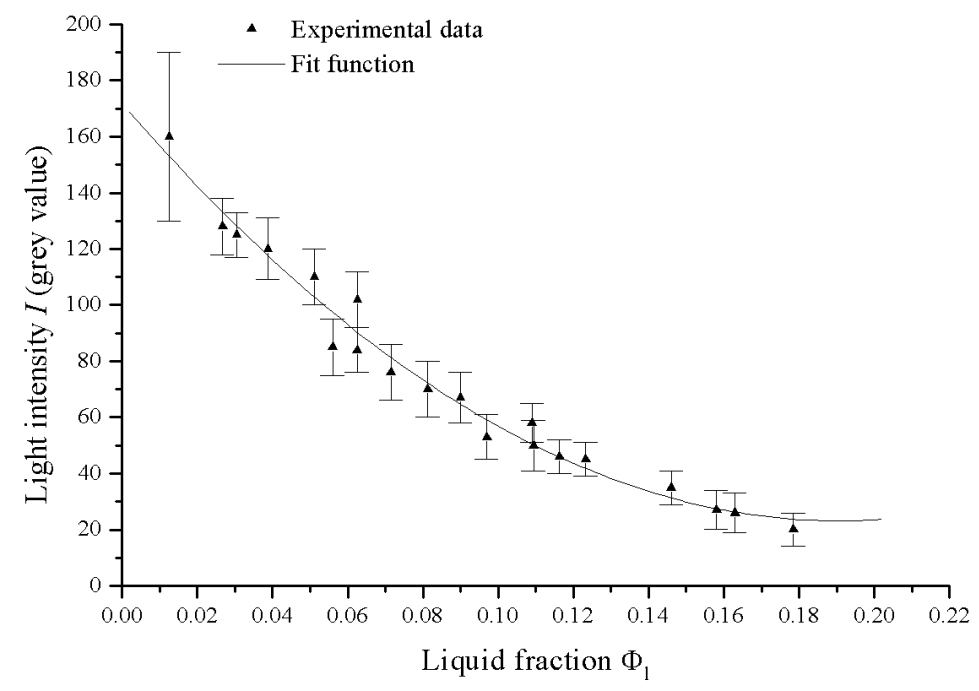

Fig. 3. The calibration relationship between liquid fraction $\Phi_{l}$ and measured light intensity $I$ is obtained from one-dimensional forced drainage experiments (see text). The empirical relationship $I=172-1559 \Phi_{l}+4081 \Phi_{l}^{2}$ provides a good description of the data. We solve this quadratic to give liquid fraction values for the experiments.

It is a partial differential equation for the cross-sectional area $A$ of the Plateau borders (which is a measure of the liquid fraction) as a function of time $t$ and vertical and horizontal coordinates $z$ (in the direction of gravity) and $x$. The differential operator is $\nabla=(\partial / \partial x, \partial / \partial z)$.

The physical parameters are the liquid density $\rho$, liquid viscosity $\mu$, surface tension $\gamma$, and acceleration due to gravity $g$. The geometrical constant $C=\sqrt{\sqrt{3}-\pi / 2}$ is related to the shape of the Plateau borders [1].

In order to solve eqn.(2) numerically we re-write it in terms of dimensionless quantities by introducing the length-scale $x_{0}=2^{7 / 12} 12^{-1 / 2} V_{b}^{1 / 3}=0.433 V_{b}^{1 / 3} ; V_{b}=4 \pi / 3(d / 2)^{3}$ is the bubble volume, so that $x_{0}$ is a measure of the length of each Plateau border if the structure were that of a repeated Kelvin cell [1,11]. We choose a dimensionless Plateau border area of $\alpha=A / x_{0}^{2}$, so that the liquid fraction $\Phi_{l}$ is numerically equal to $\alpha$. The time-scale is $t_{0}=150 \mu x_{0} /(C \gamma)$ and we introduce a Bond number $B o=\rho g x_{0}^{2} /(C \gamma)$ to measure the relative importance of gravitational over surface tension dominated flow [6]. Denoting the vertical and horizontal coordinates by $\xi=z / x_{0}$ and $\eta=x / x_{0}$ respectively, we obtain

$$
\frac{\partial \alpha}{\partial \tau}+\frac{\partial}{\partial \xi}\left(B o \alpha^{2}-\frac{\sqrt{\alpha}}{2} \frac{\partial \alpha}{\partial \xi}\right)-\frac{\partial}{\partial \eta}\left(\frac{\sqrt{\alpha}}{2} \frac{\partial \alpha}{\partial \eta}\right)=0
$$


where the terms in parentheses are the vertical and horizontal flow-rates respectively.

Note that equation (3), which we solve using a finite difference method, contains no free parameters. However, in order to compare it with experimental results it is necessary to relate the dimensionless input flow-rate $Q_{\text {sim }}$ to the flow-rate used in experiments $Q_{\text {exp }}$. This flow-rate is given by

$$
Q_{s i m}=\frac{t_{0} Q_{e x p}}{x_{0}^{2} D}
$$

which is spread over a width $2 w / x_{0}$. We choose an initial uniform liquid fraction for the dry foam of $5 \times 10^{-3}$ to match the experiments.

\section{Results}

As shown in figure 2, the draining liquid spreads both horizontally and vertically after entering the foam from a narrow input (a single needle, much narrower than the width of the cell, giving a value of $w$ close to $0.15 \mathrm{~cm}$ ). The questions which we want to address concern the velocities at which this spreading takes place, especially their dependence on the width of the inlet with respect to the width of the cell.

Using the experimental technique described in section 2 we have monitored the liquid fraction along the vertical line down the centre of the cell and along a horizontal line a distance $1 \mathrm{~mm}$ below the top of the foam. An example is shown in figure 4, together with numerical solutions of eq. (3). The propagation of the drainage wave can then be tracked by measuring the position at which a particular value of liquid fraction is attained along each line, as indicated by the dotted line in figure 4 , to give the data shown in figure 5 .

\subsection{Small input width}

The data shown in figures 4 and 5 concerns an experiment for which the flow-rate was $Q_{\exp }=$ $0.005 \mathrm{ml} / \mathrm{s}$ and the input half-width $w=0.15 \mathrm{~cm}$.

These profiles of liquid fraction are for the very beginning of the propagation of the drainage wave, and show the initial build-up of liquid at the top of the foam and the beginnings of the more familiar drainage wave. It is difficult to achieve a full profile of the wave in our relatively small 
(a)

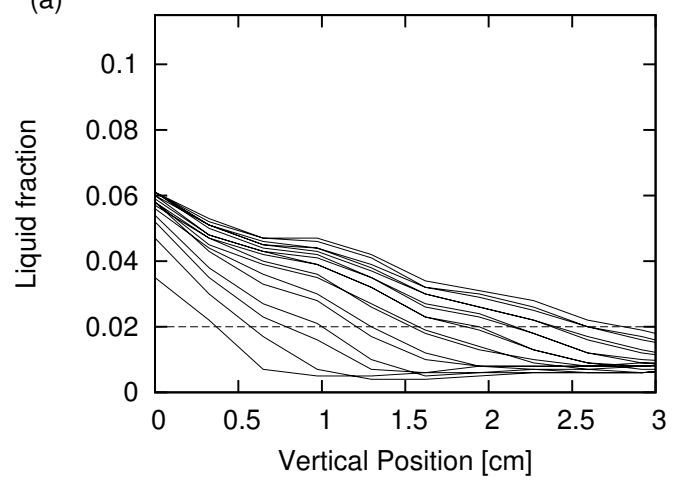

(c)

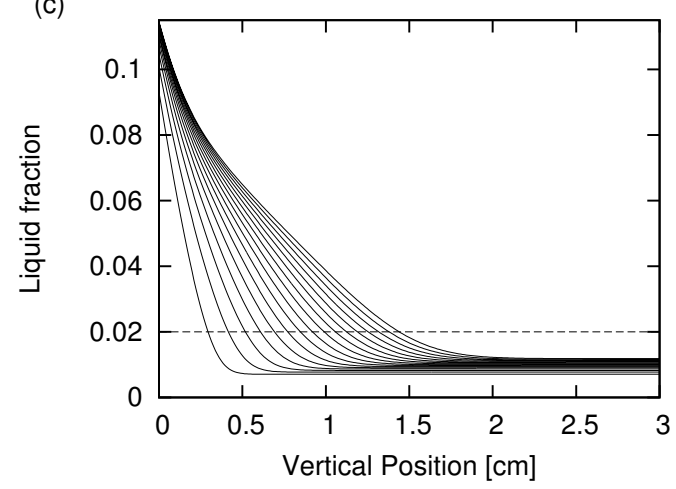

(b)

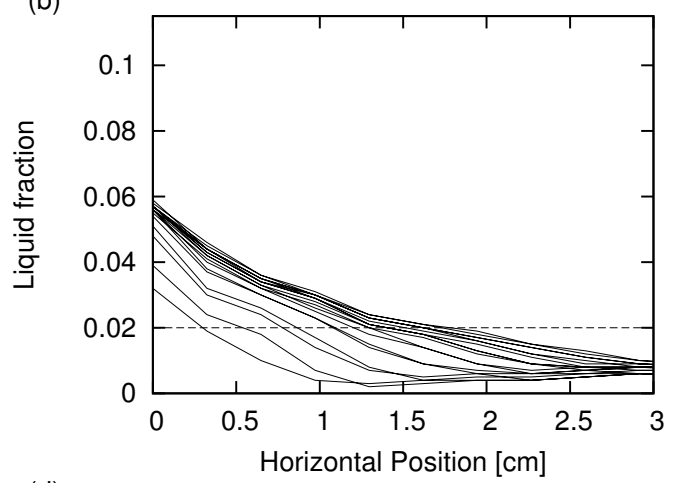

(d)

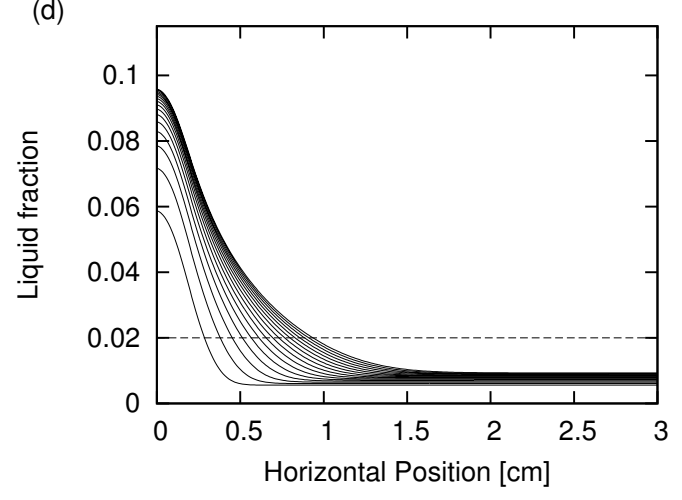

Fig. 4. Profiles of liquid fraction along the vertical and horizontal lines indicated in figure 2. The results of experiment for the vertical (a) and horizontal (b) directions show qualitative agreement with those from simulation, (c) and (d). In this case the input flow-rate was $Q_{\text {exp }}=0.005 \mathrm{ml} / \mathrm{s}$ over a width $w=0.15 \mathrm{~cm}$. Time increases in equal increments of $0.85 \mathrm{~s}$.

experimental apparatus because of the time taken to fill the cell with monodisperse foam: diffusive coarsening becomes noticeable after about 30 minutes and limits the size of the foam samples that can be used.

The results show that the variation of the position of the wave-front in the vertical direction is well described by a power-law, $z \propto t^{\beta}$. The exponent in this case is $\beta=0.67$. Thus the velocity of the wave decreases as it descends and spreads out.

For the numerical simulation corresponding to this experiment, length and time scales are set by $x_{0}=0.35 \mathrm{~mm}$ and $t_{0}=0.0053 \mathrm{~s}$ and the Bond number is $B o=0.12$. In this case we find $\beta=0.69$ which compares well with the experimental value. The profiles of liquid fraction (figure 4, a and c) also show qualitative agreement, to the extent that they show the same hump a short distance from the input where the true wave starts to form. However, the simulation evidently overestimates the liquid fraction and underestimates the velocity of the wave, each by a factor close to two as in the one-dimensional case [1].

Note that unlike the situation in one dimension, here the exponent is slightly dependent on the 

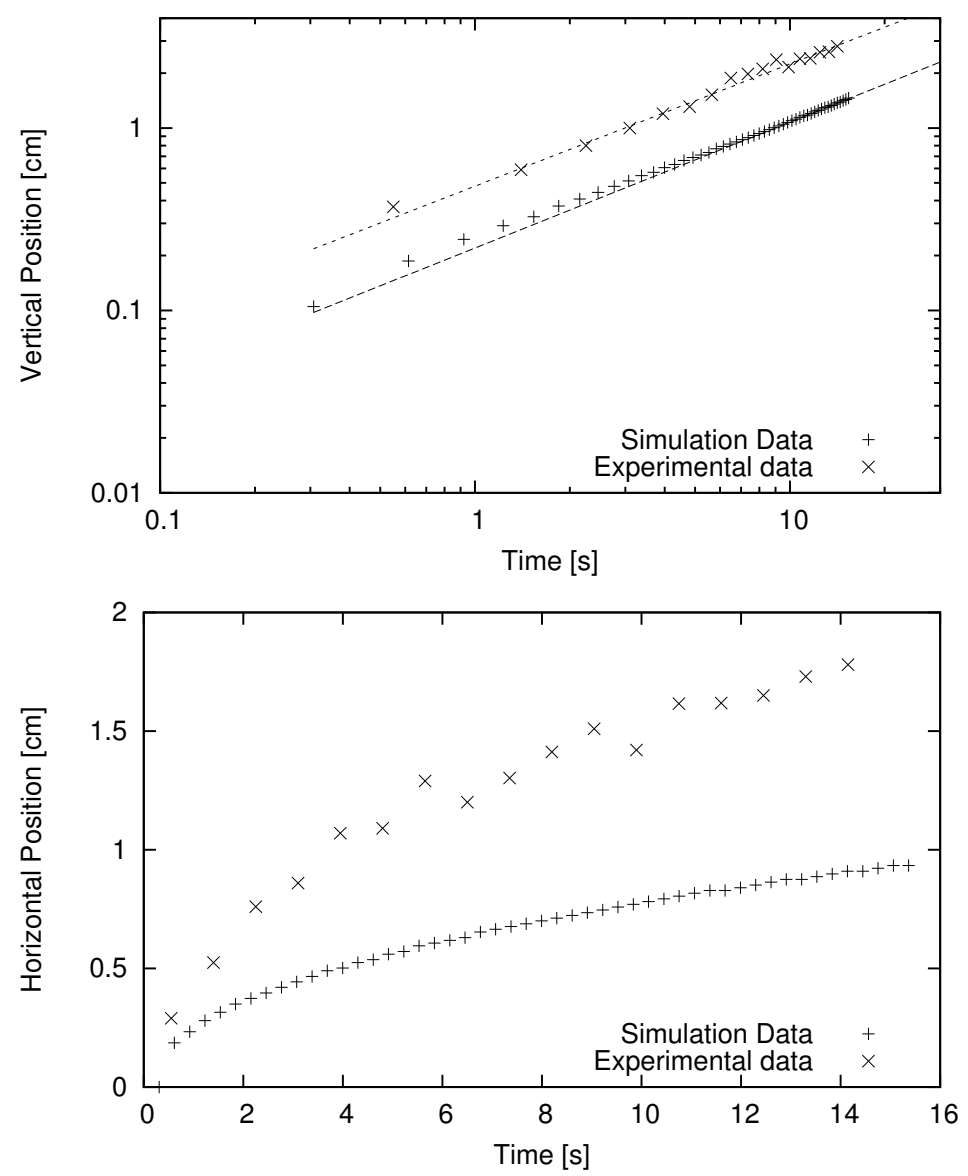

Fig. 5. The position of the wave-front in both the vertical (upper) and horizontal (lower) directions, as a function of time. The results of simulation and experiment are shown for the case of a flow-rate of $Q=0.005 \mathrm{ml} / \mathrm{s}$ and $w=0.15 \mathrm{~cm}$. The vertical position is shown on a double logarithmic scale, indicating that a power-law describes the data well. To find the exponent of this power-law, we fit only the data for $t>4 \mathrm{~s}$. The horizontal velocity of the wave cannot be described by a power-law in this way.

value of liquid fraction that is tracked. We find a variation of up to $10 \%$ in the exponent when the liquid fraction at which the front is measured changes from a value close to the background (initial) liquid fraction of 0.005 up to about 0.05 .

The situation is different for the propagation in the horizontal direction (figure $4, \mathrm{~b}$ and $d$ ). A power-law no longer describes the front position as it spreads across the foam (figure 5). We will investigate this horizontal spreading in future work, but note that the agreement between simulation and experiment is good. 

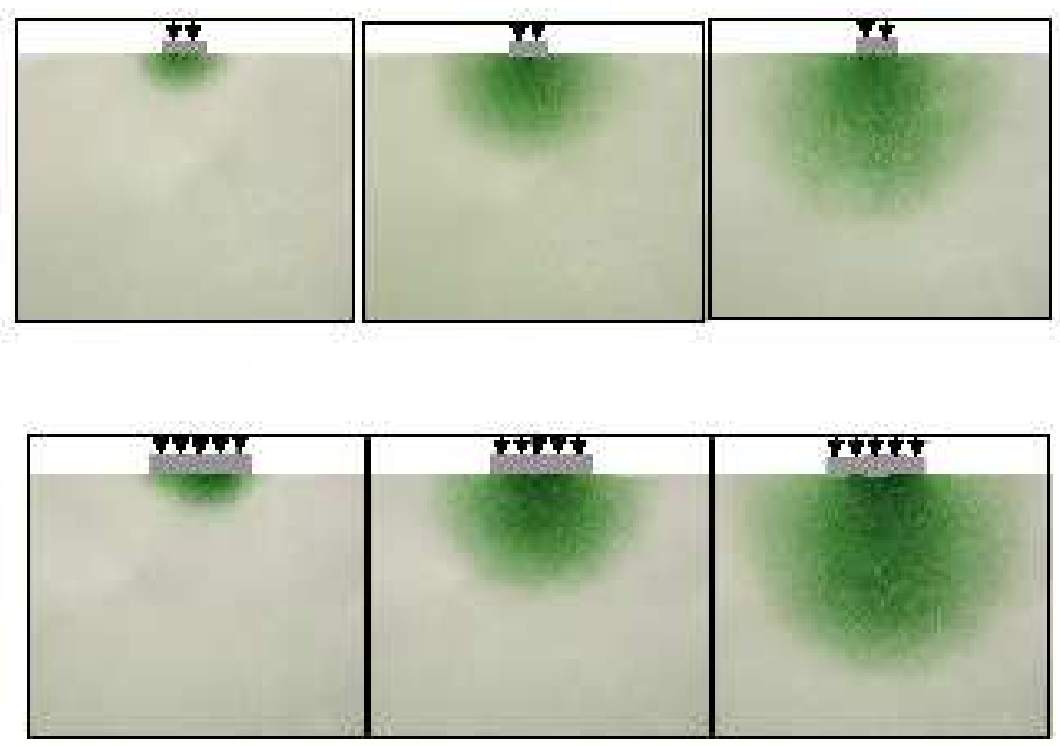

Fig. 6. In these experiments with a wider input, it is clear that the drainage wave spreads more quickly in the vertical direction and the opposite happens in the horizontal direction. Images are shown after 1, 7 and 14 seconds for input-widths which are twice (top row) and five-times (bottom row) the value shown in figure 2. In each case we track the positions of the wetting fronts in both the vertical and horizontal directions. The results are shown in figure 7.

\subsection{Varying input width}

We have repeated the above experiments for a range of input widths $w$, keeping the dimensions of the Hele-Shaw cell and the bubble size constant, as illustrated in the photographs of figure 6 . This results in an increase of the exponent describing the vertical velocity of the drainage wave, shown in figure 7 . As the width of the input increases, the wave moves faster, and slows down less as it descends. We expect this exponent to tend smoothly to one as $w \rightarrow W$, corresponding to a "one-dimensional" solitary wave with no effective horizontal motion. The results for the exponents from the corresponding simulations are consistent with this, and as figure 7 shows, are in good agreement with the experimental results.

\section{Conclusion}

The reasonable agreement between the power-law exponents taken from experiment and simulation increases our confidence in the predictive capability of the drainage equation appropriate for Poiseuille flow. The discrepancy in the prefactors (corresponding to the liquid fraction being over- 


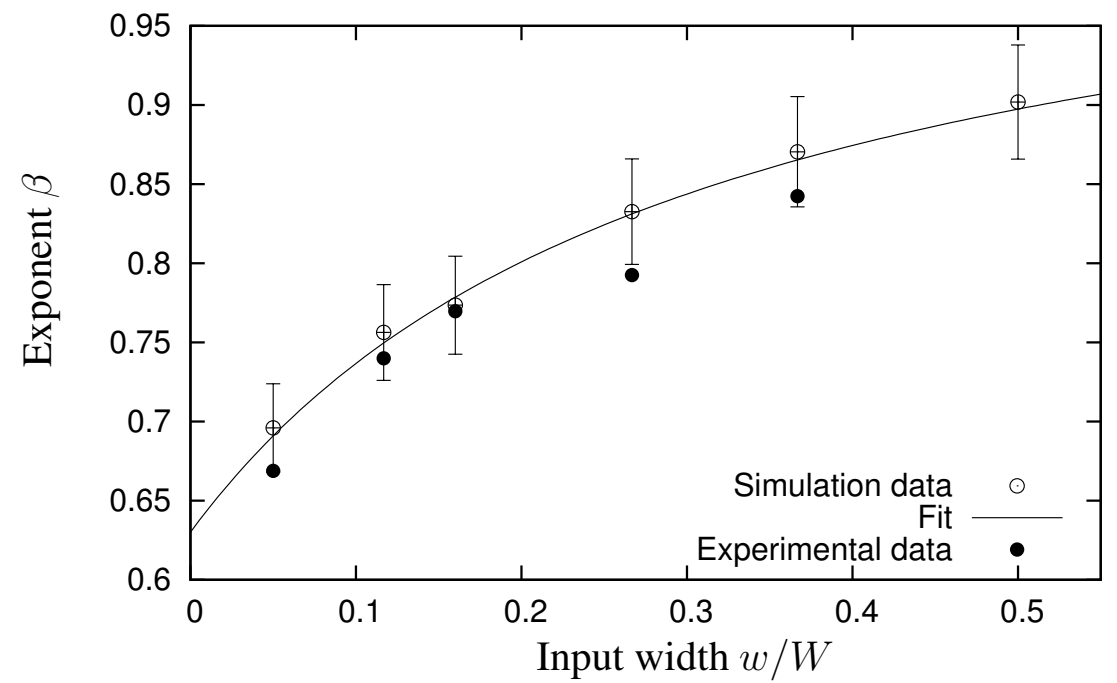

Fig. 7. The influence of the input width on the motion of the drainage wave in the vertical direction, comparing experimental measurements and numerical simulations. The exponent $\beta$ in the power-law $z \propto t^{\beta}$ describing the vertical velocity of the wave increases as the input width increases. Despite the discrepancy in the actual position of the wave (figure 4), the exponents found in the simulations, fitted to a function $\beta=0.63+1.42(w / W) /(1+3.31 w / W)$, show good agreement with the experiments.

estimated and the drainage velocity being underestimated in the simulations) will be investigated in future work. We will then also study drainage in non-rectangular geometries and also experiment with emulsions, which have been shown to adhere to the approximations made in deriving the drainage equation given here.

\section{Acknowledgements}

SJC thanks R. Meloy for useful discussions. This work was supported by Enterprise Ireland (EI-SC/2002/11), the European Space Agency (14914/02/NL/SH, 14308/00/NL/SH (AO-99-031) CCN 002 MAP Project AO-99-075) and the Royal Irish Academy (Royal Society Exchange Scheme).

\section{References}

[1] Weaire, D. and Hutzler, S., 1999, The physics of foams (Oxford: Oxford University Press).

[2] Banhart, J. and Weaire,D. 2002, Physics Today July: 37-42.

[3] Weaire, D., Hutzler, S., Verbist, G. and Peters, E., 1997, Adv. Chem. Phys 102, 315-374.

[4] Koehler, S.A., Hilgenfeldt, S. and Stone, H., 2000, Langmuir 16 6327-6341. 
[5] Leonard, R.A. and Lemlich, R., 1965, A.I.Ch.E. J. 11 18-29.

[6] Koehler, S.A., Hilgenfeldt, S. and Stone, H.A., 2001, Europhys. Lett. 54, 335-341.

[7] Grassia, P., Neethling, S.J. and Cilliers, J.J., 2002, Euro. Phys. J. E 8 517-529.

[8] Durand, M., Martinoty, G., Langevin, D., 1999 Phys. Rev. E. 60 R6307-R6308.

[9] Hutzler, S., Cox, S.J., Weaire, D. and Wilde, P.J., 2000 in Foams, emulsions and their applications, eds. Z. Pacelli, J. Banhart and G. Verbist, Verlag MIT, Bremen, 5-12.

[10] Cox, S.J., Weaire, D., Hutzler, S., Murphy, J., Phelan, R. and Verbist, G., 2000, Proc. R. Soc. Lond. A 456, 2441-2464.

[11] Cox, S.J. and Verbist, G., 2003, Microg. Sci. Tech. XIV/4, 45-52. 